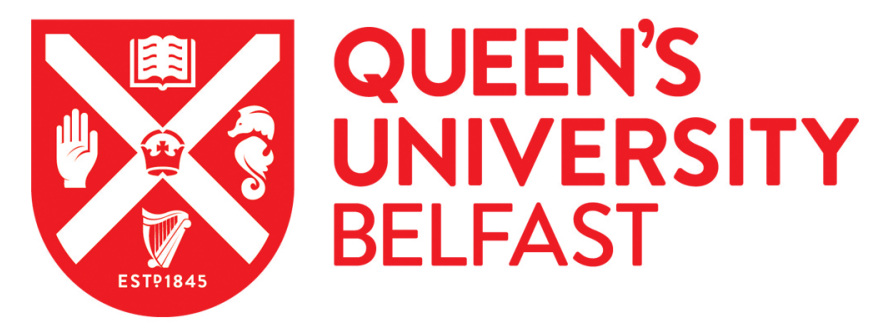

\title{
Charge steering of laser plasma accelerated fast ions in a liquid spray - Creation of MeV negative ion and neutral atom beams
}

Schnürer, M., Abicht, F., Prasad, R., Borghesi, M., Priebe, G., Braenzel, J., Andreev, A., Nickles, P. V., Jequier, S., Tikhonchuk, V., \& Ter-Avetisyan, S. (2013). Charge steering of laser plasma accelerated fast ions in a liquid spray - Creation of MeV negative ion and neutral atom beams. Physics of Plasmas, 20(11), [113105]. https://doi.org/10.1063/1.4829005

Published in:

Physics of Plasmas

Document Version:

Publisher's PDF, also known as Version of record

Queen's University Belfast - Research Portal:

Link to publication record in Queen's University Belfast Research Portal

Publisher rights

CC-BY

\section{General rights}

Copyright for the publications made accessible via the Queen's University Belfast Research Portal is retained by the author(s) and / or other copyright owners and it is a condition of accessing these publications that users recognise and abide by the legal requirements associated with these rights.

Take down policy

The Research Portal is Queen's institutional repository that provides access to Queen's research output. Every effort has been made to ensure that content in the Research Portal does not infringe any person's rights, or applicable UK laws. If you discover content in the Research Portal that you believe breaches copyright or violates any law, please contact openaccess@qub.ac.uk. 


\section{AIP | Physissof of}

Charge steering of laser plasma accelerated fast ions in a liquid spray - creation of $\mathrm{MeV}$ negative ion and neutral atom beams

M. Schnürer, F. Abicht, R. Prasad, M. Borghesi, G. Priebe, J. Braenzel, A. Andreev, P. V. Nickles, S. Jequier, V. Tikhonchuk, and S. Ter-Avetisyan

Citation: Physics of Plasmas (1994-present) 20, 113105 (2013); doi: 10.1063/1.4829005

View online: http://dx.doi.org/10.1063/1.4829005

View Table of Contents: http://scitation.aip.org/content/aip/journal/pop/20/11?ver=pdfcov

Published by the AIP Publishing

$\underset{\substack{\text { Aubloning } \\ \text { sil }}}{A}$ Re-register for Table of Content Alerts 


\title{
Charge steering of laser plasma accelerated fast ions in a liquid spray - creation of MeV negative ion and neutral atom beams
}

\author{
M. Schnürer, ${ }^{1}$ F. Abicht, ${ }^{1}$ R. Prasad,${ }^{2}$ M. Borghesi,${ }^{3,4}$ G. Priebe, ${ }^{1}$ J. Braenzel, ${ }^{1}$ \\ A. Andreev, ${ }^{1,5}$ P. V. Nickles, ${ }^{6}$ S. Jequier, ${ }^{7}$ V. Tikhonchuk, ${ }^{7}$ and S. Ter-Avetisyan ${ }^{4}$ \\ ${ }_{1}^{1}$ Max Born Institute for Nonlinear Optics and Short Pulse Spectroscopy, 12489 Berlin, Germany \\ ${ }^{2}$ Institute for Laser and Plasma Physics, Heinrich Heine University, Duesseldorf 40225, Germany \\ ${ }^{3}$ School of Mathematics and Physics, The Queen's University of Belfast, Belfast BT7 INN, United Kingdom \\ ${ }^{4}$ ELI-Beamlines, Institute of Physics, Czech Academy of Science, 18221 Prague, Czech Republic \\ ${ }^{5}$ Vavilov State Optical Institute, 119034 St. Petersburg, Russia \\ ${ }^{6}$ WCU Department of Nanobio Materials and Electronics, Gwangju Institute of Science and Technology, \\ Gwangju, South Korea \\ ${ }^{7}$ Centre Lasers Intenses et Applications, University of Bordeaux, CEA, CNRS, 33405 Talence, France
}

(Received 14 October 2013; accepted 20 October 2013; published online 12 November 2013)

\begin{abstract}
The scenario of "electron capture and loss" has been recently proposed for the formation of negative ion and neutral atom beams with up to $\mathrm{MeV}$ kinetic energy [S. Ter-Avetisyan, et al., Appl. Phys. Lett. 99, 051501 (2011)]. Validation of these processes and of their generic nature is here provided in experiments where the ion source and the interaction medium have been spatially separated. Fast positive ions accelerated from a laser plasma source are sent through a cold spray where their charge is changed. Such formed neutral atom or negative ion has nearly the same momentum as the original positive ion. Experiments are released for protons, carbon, and oxygen ions and corresponding beams of negative ions and neutral atoms have been obtained. The electron capture and loss phenomenon is confirmed to be the origin of the negative ion and neutral atom beams. The equilibrium ratios of different charge components and cross sections have been measured. Our method is general and allows the creation of beams of neutral atoms and negative ions for different species which inherit the characteristics of the positive ion source. (C) 2013 Author(s). All article content, except where otherwise noted, is licensed under a Creative Commons Attribution 3.0 Unported License. [http://dx.doi.org/10.1063/1.4829005]
\end{abstract}

The interest in negative ions in fundamental science and technology has increased substantially in recent years. The applications of negative ion beams range from use in surface growth and deposition processes to elucidating the details of chemical reactions under controlled collisions. In many upcoming structural processes, including implantation for micrometer-sized powder surface modification, ${ }^{1}$ damage-free nanoparticle formation for quantum devices in a thin insulator film, ${ }^{2}$ or the so-called bio-compatibility surface treatment in nerve cell engineering, ${ }^{3,4}$ utilisation of negatively charged ions shows very promising results. The possibility of easy neutralization of negative ions is exploited in the accelerator technology, including injectors dedicated to heating of tokamak plasmas (e.g., the negative-ion-based Neutral Beam Injection (NBI) system in the International Thermonuclear Experimental Reactor (ITER) ${ }^{5,6}$ ) and the next generation of particle accelerators such as spallation sources (ESS). ${ }^{7,8}$ Additionally, negative ions have been proposed recently as an alternative to positive ions for heavy ion fusion drivers in inertial confinement fusion, because negative ions prevent electron accumulation and, if desired, the beams could be photo detached to neutrals. All these applications are demanding a sufficiently high flux of negative ions from different atomic species and over a wide range of energies. The creation of neutral atom beams is generally coupled to the negative ion beam technology due to their easy neutralisation.

In the present paper we are demonstrating how the new achievements in positive ion source technology associated to intense laser-matter interactions can be directly transferred to negative ion and neutral atom beams. Our method is very general and allows production of a broad variety of neutral atoms and negative ions with $\mathrm{MeV}$ energies.

In general, while several types of positive ion sources are readily available for different applications, the negative ion source development has generally suffered stronger constraints, because handling of such a loosely bound system as a negative ion is a technological challenge. Several methods for negative ion beam production have been developed during the past few decades: (i) The double-charge-exchange method was the one employed in early 1960s to produce negative ions. A beam of positive ions accelerated to several $\mathrm{keV}$ was passed through a charge-exchange medium (e.g. a gas cell or jet) and a small fraction of it collects two electrons and becomes negatively charged. In this, sources of negative ions utilising the charge-exchange phenomena in alkali metal vapours. ${ }^{9,10}$ (ii) In volume devices, negative ions are generated in discharges due to electron-neutral collisions at a low energy. ${ }^{11,12}$ The creation of negative ions is based on the polarization of the atom induced by an approaching electron. A biased grid set is used to extract negative ions from the discharge exit orifice. (iii) The surface-plasma negative ion sources are based on positive ion bombardment of an emitter surface. ${ }^{13,14}$ Here the main mechanism of negative ion formation is the secondary emission of sputtered and scattered particles accompanied by the capture of electrons from the electrodes. 
Our approach discussed here is completely different: it is based on changing the charge state of different species of already accelerated positive ions. They interact with a liquid spray $^{15}$ and are transformed into corresponding negative ions or neutral atoms while keeping their energy and momentum nearly unchanged.

Negative ions have been observed recently in a number of laser plasma experiments. Emission of $\mathrm{C}^{-}$ions from a laser heated $\mathrm{CO}_{2}$ cluster target was reported in Ref. 16 where Coulomb implosion was suggested as a mechanism of negative ion acceleration in the direction opposite to that of positive ions. In laser-plasma interaction experiments an energetic $\mathrm{O}^{-}$ion emission was found from a water spray target, ${ }^{17}$ and on the basis of experimental findings the electron capture and loss mechanisms were proposed to explain the observations. Laser accelerated negative ions and neutral atoms of argon from clusters were reported in Ref. 18 where observations were also ascribed to the charge exchange process.

The proposed "electron capture and loss" scenario ${ }^{17}$ suggests that independently from the location of the positive ion source the capture or loss of an electron occurs while a fast positive ion passes through the spray. Therefore, a separation of the ion source and the spray, i.e., the acceleration of positive ions from a foil target followed by the passage through a cold spray, should also result in the appearance of negative ions and neutral atoms if the scenario proposed in Ref. 17 is correct. Additionally, with this approach a complicated nature of the ion acceleration phenomena, such as temporal, spatial, and characteristics of electrons and ions within the laser plasma source can be kept out of consideration. The electron capture and loss mechanisms can thus be studied separately and the real origin of negative ions and neutrals can be unambiguously identified.

The experimental arrangement is presented in Fig. 1. The ion beam is accelerated by irradiating $5 \mu \mathrm{m} \mathrm{CHO} \mathrm{or} \mathrm{Ti}$ foil targets with $40 \mathrm{fs}, 1 \mathrm{~J}$ Ti:Sapphire laser pulses at an intensity of $5 \times 10^{19} \mathrm{~W} / \mathrm{cm}^{2}$. A well characterised either water ${ }^{15}$ or ethano ${ }^{19}$ spray positioned at the distance of $4 \mathrm{~cm}$ from the foil target allowed us to study the ion-spray interaction.

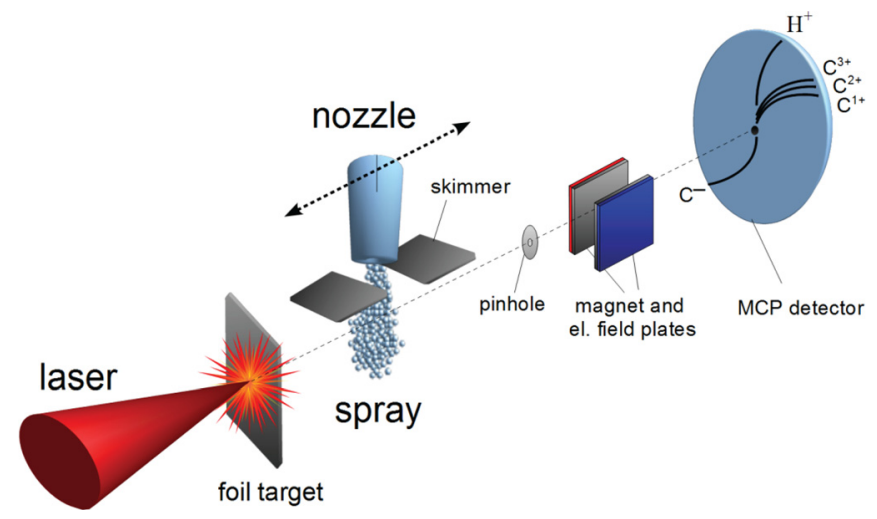

FIG. 1. Experimental setup for investigation of electron capture and loss processes during the passage of fast positive ions through a liquid spray target. Laser accelerated, fast positive ions from a foil target propagated through a cold spray. The double ended arrow indicates that the spray position between target foil and spectrometer could be changed. A Thomson spectrometer, with parallel electric and magnetic fields measures ion energy distributions.
A Thomson spectrometer enabled absolute measurements of both positive and negative ions, in a single shot. ${ }^{20,21}$ Particles with opposite charges are deflected in opposite directions in the magnetic and electric fields of the spectrometer thus creating oppositely directed spectral traces on the detector screen (a Micro Channel Plate, MCP). The position of the particle on the spectral trace depends on its energy: the higher is the ion energy less it is deviated from the zero point where undeflected emissions: $x$-rays and neutral atoms hit the detector. Typically, a magnetic field of about $0.27 \mathrm{~T}$ and an electric field of $2-4 \mathrm{kV} / \mathrm{cm}$ have been applied and ions have been detected through a $200 \mu \mathrm{m}$ pinhole.

The interaction region, i.e. the length, of the spray was up to $2 \mathrm{~mm}$ and consisted of droplets with a diameter of $150 \mathrm{~nm}$ in the case of water $\left(\mathrm{H}_{2} \mathrm{O}\right)$ or $180 \mathrm{~nm}$ in the case of ethanol $\left(\mathrm{C}_{2} \mathrm{H}_{5} \mathrm{OH}\right)$ sprays. The average molecular densities are $2 \times 10^{18}$ and $\sim 10^{19} \mathrm{~cm}^{-3}$ for the water and ethanol, respectively. In the experiments the width of the spray and thus the propagation length of ions through the spray were controlled using a skimmer.

Accelerated ion beams from the foil target at different laser shots are found to be identical within $\pm 5 \%$ if they have the same cut off energy of protons although the spectral shapes could be slightly different. The liquid spray is transparent for protons with energies down to a few hundred $\mathrm{keV}$ (the proton energy loss in the water spray is less than $50 \mathrm{keV} / \mathrm{mm}$ ). Thus the proton signal can serve as a reference to verify that in different laser shots similar ion beams have been produced, which was fundamental for the comparison of ion spectra obtained with and without the spray. The measurements of the energy distribution of proton and $\mathrm{C}^{\mathrm{q}+}$ beams (q represents the fast ion charge) without spray and after interaction with a spray have been accomplished in a single laser shot. Typical CCD pictures of ion spectra from the phosphorous screen of the MCP are shown in Fig. 2. Here protons, carbon, and oxygen ions are accelerated from polymer $\mathrm{CHO}$ foil target. The corresponding spectra deduced from these digital images are shown in Fig. 3.

In these shots the measured proton spectral distributions without spray and when they have passed through the spray are quite similar (Fig. 3(a)) and protons have the same cutoff energy, which, as explained above, is an indicator that the carbon ion spectra produced from the source were also very similar in the two events. In contrast, a substantial redistribution of carbon ion charge states (compare Figs. 3(b) and $3(\mathrm{c}))$ is recorded and, most remarkably, new charge species, the $\mathrm{C}^{-}$and $\mathrm{O}^{-}$ions, appeared when carbon and oxygen ions propagated through the spray (Figs. 3(c) and 3(d)).

Since the propagation of carbon and oxygen ions through a spray is highly collisional (with mean free path length is less than $50 \mu \mathrm{m}$ ), the measured strong modification of ion spectra and appearance of $\mathrm{C}^{-}$and $\mathrm{O}^{-}$ions can be assigned to the electron capture and loss processes during ion propagation through the spray. If no interaction with the spray occurs (spray generator is off) the cut off energies of carbon ions increase with the ion charge state (Fig. 3(b)). On the contrary, after an interaction with the spray $\mathrm{C}^{\mathrm{q}+}$ ions have similar cut-off energies, which shows that the recombination from the $\mathrm{q}+1$ to the $\mathrm{q}$ state occurs without losing 

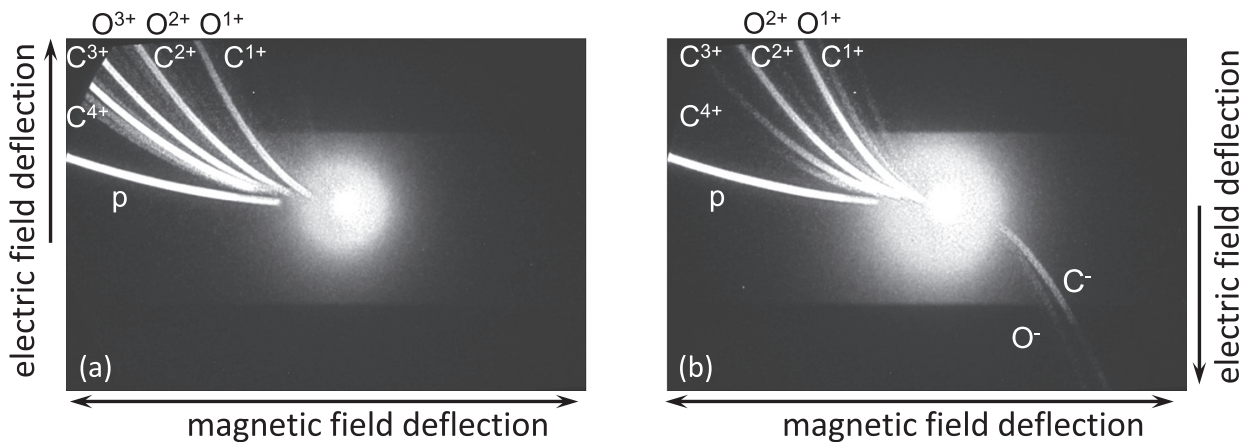

FIG. 2. Thomson-parabola traces of carbon ion and proton spectra (a) accelerated from carbon foil target and (b) accelerated from carbon foil target and propagated through the water spray. From the foil target only positive carbon and oxygen ions with the charge states $1+$ up to $4+$ and $\mathrm{H}^{+}$are visible. The bright circle at the centre of the image is formed by neutrals and energetic photons moving along the axis of the spectrometer. When the ion beam was propagating through the spray, its charge distribution is changed and $\mathrm{C}^{-}$and $\mathrm{O}^{-}$ions appear.

energy (Fig. 3(c)). Moreover, the relative weight of the charge state distributions has been changed as compared to the ratios observed in the original spectrum (from the foil) while traversing the spray. In particular, the original feature of the ion spectrum exhibiting an increase of energy cutoff values with the charge state (Fig. 3(b)) was lost. Ions recombine and their charge states have been redistributed according to the probabilities of electron capture and loss processes inside the spray. The process also results in the appearance of ions with a lower charge state and higher energies than in the original spectrum (compare Figs. 3(b) and 3(c)). In particular, the fastest $\mathrm{C}^{+}$and $\mathrm{C}^{2+}$ ions are, in fact, the ions with an initially higher charge that have captured electrons while conserving their energy. Thus, the $\mathrm{C}^{-}$ions are formed.

The $\mathrm{O}^{\mathrm{q}+}$ ion spectra have been modified similarly as discussed above, and $\mathrm{C}^{-}$and $\mathrm{O}^{-}$ions have appeared (through the same process) when the original ion beam was passed through the spray (Fig. 3(d)). The redistribution of carbon and oxygen ions charge states occurred similarly with a slightly different probability which is connected to differences in the relevant recombination cross sections.

The electron capture and loss scenario of negative ions formation implies also the existence of fast neutral atom beams. Indeed, energetic beams of hydrogen $\left(\mathrm{H}^{0}\right)$ and oxygen $\left(\mathrm{O}^{0}\right)$ atoms have been measured in recent experiments along with $\mathrm{O}^{-}$and negative hydrogen $\left(\mathrm{H}^{-}\right)$ions. ${ }^{22}$ Although the neutrals could not be directly measured in this experiment together with ions, the spectrum of neutral carbon atoms $\left(\mathrm{C}^{0}\right)$ can be deconvolved by subtraction of total number of ions measured without spray and with spray for each energy bin, as shown in Fig. 3(c). The low energy cut (below $0.4 \mathrm{MeV}$ ) is related to the limited size of the detector. The ion spectra were cut at different low energies depending on their charge.

The process of interaction of ion beam with the spray comprises the fact that ions can change their charge state several times while traversing the converter medium. As the spray is highly collisional for carbon and oxygen ions, after

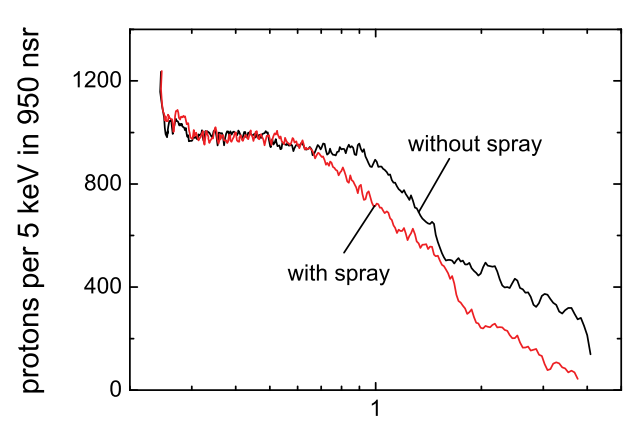

(a)

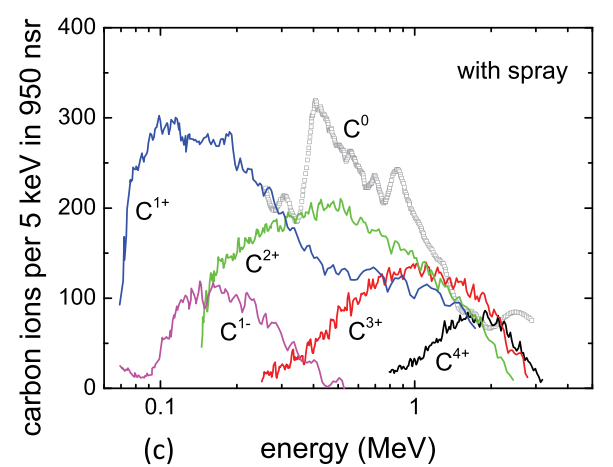

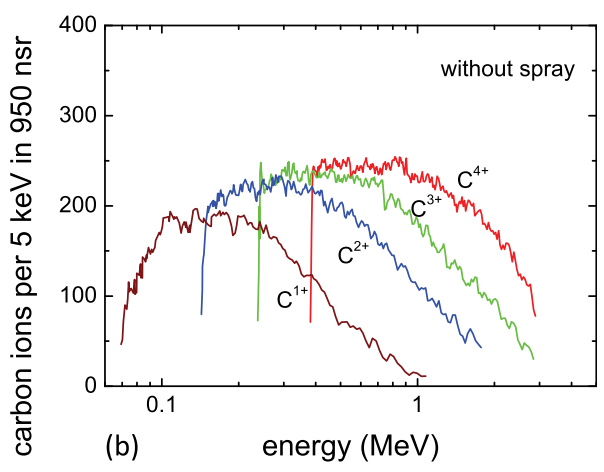

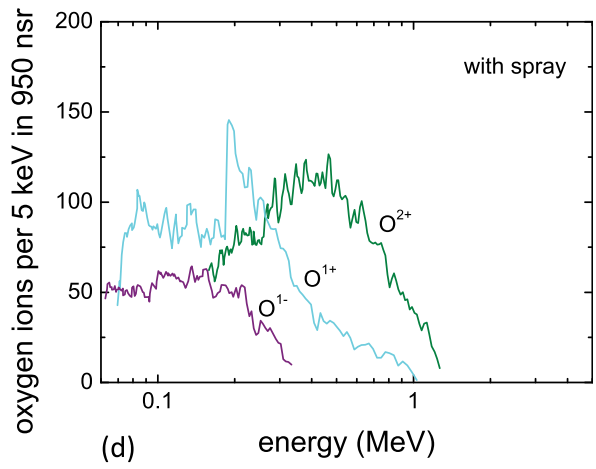

FIG. 3. Ion spectra deduced from digital images shown in Fig. 2. The proton spectral distributions are similar (a), which guarantees identical heavy ion beams accelerated in these shots. (b) Without spray, the carbon ions cut off energies and densities are increasing with increase of the ion charge state. (c) With the spray in place, these ions maintain a similar energy while they recombine to give different charge states, while their densities have been redistributed according to the probabilities of electron capture and loss by ions inside the spray. The appearance of $\mathrm{C}^{-}$and $\mathrm{O}^{-}$ions has to be noted. 
a sufficient number of collisions an equilibrium distribution of charge states can be established. This depends on the effective cross sections and the areal density of the converter-target (spray). The equilibrium-charge-state distribution is independent of the initial distribution of charge states. Each chargestate constituent in the beam reaches a certain equilibrium value which does not change when the convertor-target thickness is further increased. This was confirmed by varying the spray thickness from 0 up to $2 \mathrm{~mm}$ (fully open) using a skimmer. The number of negative ions starts decreasing only for the spray thicknesses smaller than $0.1 \mathrm{~mm}$.

Better understanding of electron capture and loss processes requires a more thorough discussion. The equilibriumcharge-state distributions are not sufficient to infer the absolute cross-sections, although certain ratios and trends of the crosssections may be deduced. For example, a strong enhancement of low charge state densities may indicate a contribution of multiple electron capture or loss processes. Moreover, distortions of the distribution in certain energy regions may originate from the shell effects in the electronic structure of the projectile ion and/or target atoms. Additionally, the excitation of the atoms in the spray prior to a charge-changing collision may influence the probabilities of electron capture and loss in subsequent collisions as suggested recently ${ }^{18}$ as an explanation for observations of negative Ar ions.

The cross sections are very complex quantities and it is rather difficult to provide an adequate quantitative description $^{23}$ of all the processes involved. A sufficiently accurate direct experimental measurement of charge distributions may define most of the parameters included in theory. For instance, we evaluated the hypothesis ${ }^{18}$ that the excitation of atoms by fast electrons and $\mathrm{x}$-rays emitted from the laser plasma source might affect the charge exchange rate. We compared the ion yield with the spray-convertor when the spray was placed at distances of $22 \mathrm{~mm}$ and $380 \mathrm{~mm}$ far from the laser plasma source. An increase of the distance between the source and the spray by a factor of 17 reduces the intensity of plasma emission (x-rays, laser light and fast electrons) by a factor of about 300. Consequently, a substantial reduction of the excited atoms is expected. However, the measured ion yield was independent on the spray distance from laser plasma ion source. Therefore, the role of excited atoms is negligible in our experiments.

The quasi-classical analysis of the capture and loss of an electron in collisions of a fast ion with atoms at rest ${ }^{24}$ suggests that the maximum cross section corresponds to the projectile velocity comparable with the orbital electron velocities $v_{0}\left(v_{0}=e^{2} / \hbar\right.$ is the Bohr velocity) of an atom in rest. These charge-exchange processes proceed elastically, without energy exchange and the angular diagram is very narrow, of the order of $1^{\circ} .^{25}$

If the cross sections of the electron capture and loss processes would be known, the relative fraction of ions in any of charge state, positive, negative, or neutral, can be predicted, and the experimental data can be explained. However the data for the cross sections of these processes reported in a literature are very limited or scattered, particularly for the energies reported here (from $100 \mathrm{keV}$ to a few $\mathrm{MeV}$ ).

The dominant charge-exchange processes are expected to be processes of one electron capture and loss:

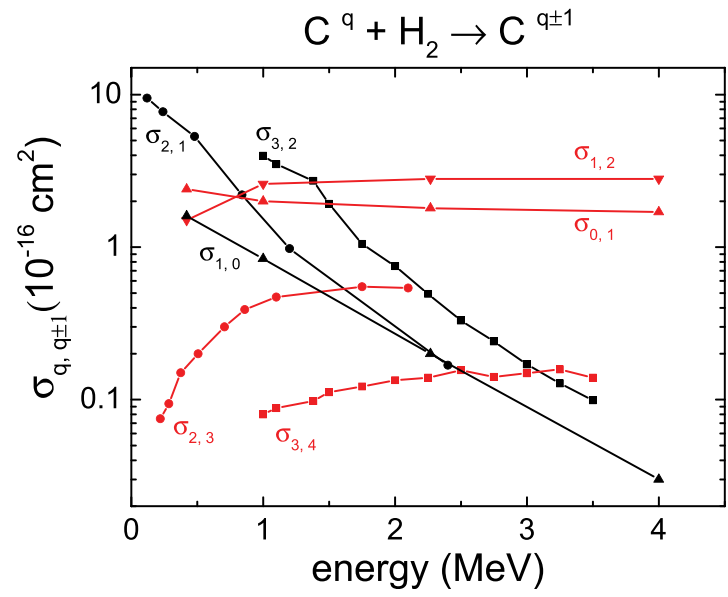

FIG. 4. Cross sections of $\mathrm{C}^{\mathrm{q}}+\left(\mathrm{H}_{2}\right) \rightarrow \mathrm{C}^{\mathrm{q} \pm 1}$ processes known from literature. ${ }^{26-29}$ Electron capture cross sections are shown in red, electron loss-in black.

$\left(A^{(q)} \leftrightarrow A^{(q \pm 1)}\right)$ with the cross sections $\sigma_{q, q \pm 1}$. The cross sections of $\mathrm{C}^{\mathrm{q}}+\left(\mathrm{H}_{2}\right) \rightarrow \mathrm{C}^{\mathrm{q} \pm 1}$ processes taken from Refs. 26-29 are shown in Fig. 4. The probabilities of electron capture or recombination are strongly decreasing at high energies while the cross sections of electron loss or further ionisation of projectile ion are slowly increasing and stays nearly unchanged above $1.5 \mathrm{MeV}$, with values above $10^{-16} \mathrm{~cm}^{2}$.

The cross section of electron capture $\mathrm{C}^{0}+\left(\mathrm{O}_{2}\right) \rightarrow \mathrm{C}^{-}$; $\sigma_{0,-1}$ is measured in the $(30-170) \mathrm{keV}$ energy interval ${ }^{30}$ being at the level of $\sim 10^{-16} \mathrm{~cm}^{2}$.

In the case of projectile oxygen $\mathrm{O}^{+}, \mathrm{O}^{0}$, and $\mathrm{O}^{-}$ions the ionisation and recombination cross sections are known for the energies up to $\sim 0.6 \mathrm{MeV},{ }^{26,31-33}$ which is lower than those discussed here. However, the general trends are quite similar to those of carbon ions at the same energies.

The variation of the charge composition of an ion beam traversing a spray target can be described by a system of coupled linear differential equations

$$
\frac{1}{n_{0}} \frac{d N_{q}}{d Z}=N_{q+1} \sigma_{q+1, q}+N_{q-1} \sigma_{q-1, q}-N_{q} \sigma_{q, q-1}-N_{q} \sigma_{q, q+1},
$$

where $N_{q}(q=-1,1,2,3,4$, and 0$)$ is the number of fast negatively and positively charged ions (of charge $q$ ) and fast neutral atoms at the output of the spray, and $n_{0}$ is the average density of the spray.

As mentioned above, the typical charge exchange cross sections are of the order of $10^{-16} \mathrm{~cm}^{-2}$ (see Fig. 4 and Refs. 26-30). Thus in a liquid spray where the average density is $>10^{18} \mathrm{~cm}^{-3}$, at the full length of $L=2 \mathrm{~mm}$, one has $n_{0} L \sigma \gg 1$. Therefore, the partial derivatives on the left hand side of Eq. (1) are practically zero. Consequently, the relative number of each species depends only on the relative cross-sections

$$
N_{q+1} \sigma_{q+1, q}-N_{q} \sigma_{q, q+1}=N_{q} \sigma_{q, q-1}-N_{q-1} \sigma_{q-1, q} .
$$

Taking into account that in the experiments the higher carbon charge state measured was $\mathrm{C}^{4+}$ one arrives at simple 


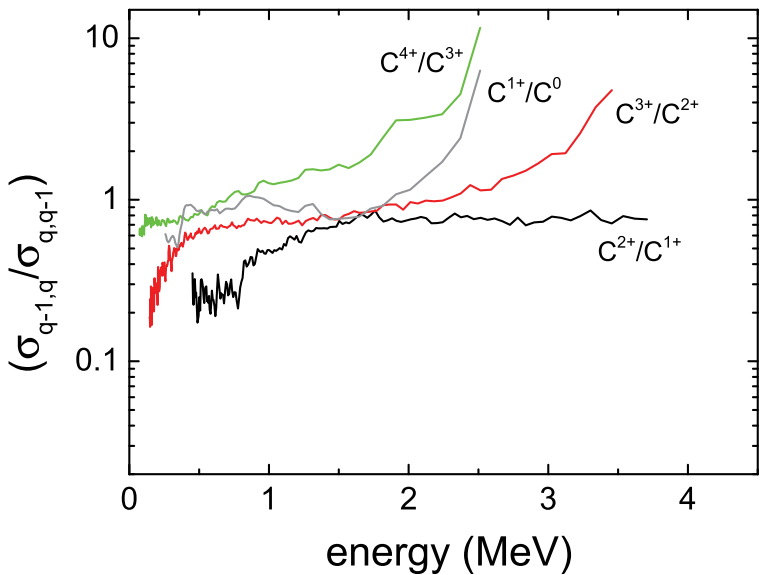

FIG. 5. The ratio of the cross sections of different carbon ions for capture and loss of a single electron deduced from Fig. 3.

relations between the number of measured ions and the cross sections as follows:

$$
\frac{N_{q}}{N_{q-1}}=\frac{\sigma_{q-1, q}}{\sigma_{q, q-1}} .
$$

These relations would be more complicated if multipleelectron capture and loss processes need to be considered. The ratios of electron capture and loss cross sections (3) for carbon ions with charge states from $\mathrm{C}^{1+}$ up to $\mathrm{C}^{4+}$ interacting with the neutral molecules in the spray are shown in Fig. 5. They are deduced from the measured ion energy spectra shown in Fig. 3.

The general trend of all measured ratios is that they are either remaining constant or increasing at high energies. Moreover, the fact that the measured ratios are higher than one in the energy range of $1-3 \mathrm{MeV}$ indicates that the electron capture or recombination cross sections are at least comparable to the corresponding electron loss or ionisation cross sections.

These trends measured in our experiment are quite different from the data available in the literature (Fig. 4), which generally show a decrease of the ratio of the corresponding recombination and ionisation cross sections with the projectile energy. In particular, a significant difference is observed for the $\sigma_{3,2} / \sigma_{2,3}$ ratio for the ion energy of $1 \mathrm{MeV}$ and for the $\sigma_{2,1} / \sigma_{1,2}$ and $\sigma_{1,0} / \sigma_{0,1}$ ratios for the ion energy of $2 \mathrm{MeV}$. Such difference between the known data and our measurements has no clear explanation at the moment. Qualitatively it is in agreement with the observation reported for argon ions in Ref. 18, however their hypothesis of the important role of excited atoms is not confirmed. It might be that in the energy range of a few $\mathrm{MeV}$ the projectile ion interacts resonantly with the electrons in the deeper atomic shells or collective excitations in the atomic clusters provide beneficial conditions for the recombination processes.

Unfortunately, the number of negative ions was below the detection threshold for the energies above $0.4 \mathrm{MeV}$. Thus the energy range of the evaluated spectrum of neutral atoms overlaps with the $\mathrm{C}^{1-}$ spectrum only in a very small interval, which does not allow to obtain the cross section ratio for $\mathrm{C}^{0}$ and $\mathrm{C}^{1-}$ ions. However, as an estimate one can take a spectrum of $\mathrm{C}^{0}$ atoms with faster growing tendency at lower energies $(<0.5 \mathrm{MeV})$ than $\mathrm{C}^{1-}$. This would suggest a cross section of electron capture by the neutral carbon atom $\left(\sigma_{0,-1}\right)$ being about one order of magnitude smaller than the cross section of electron loss by the negative ion $\left(\sigma_{-1,0}\right)$. This estimate agrees with the known ratio of the corresponding cross sections for the hydrogen ions.

Subsequent investigations are required to further elucidate the negative ion formation scenario, where special attentions have to be paid on precise measurements of the neutral particles, and their spectra, which then would be a benchmark for precise quantum mechanical calculations of the cross sections of electron capture and loss in multi-electron configurations. However, the appearance of copious energetic negative ions ${ }^{17}$ indicates that such cross sections should be relatively high.

It is clear that complex collision systems such as those studied in the present work require a sophisticated model in order to better understand the dynamics involved in the electron transfer processes. Until such a model is available, the present experiments open a possibility for measuring the ratios of the cross sections of electron capture and loss processes to benchmark future theories, and demonstrate that efficient neutralisation of carbon and oxygen ions with energies up to $1 \mathrm{MeV}$ can take place.

The demonstration of a technique for controlling the charge state and distribution of energetic positive ion beam with remarkably stable and reproducible characteristics ${ }^{15,19}$ opens unique possibilities for efficient and compact sources of energetic negative and neutral atom species of a broad variety. In the steering processes the initial positive ion beam characteristics, such as energy and laminarity are preserved. This may find many new applications in atomic physics, in controlled atom deposition technology, in controlled chemical reactions, and in accelerator technology.

Furthermore, the proposed method allows to measure cross sections for single-ionization, and single capture processes in $\mathrm{H}^{+}, \mathrm{O}^{+}$, and $\mathrm{C}^{+}+\mathrm{H}_{2} \mathrm{O}$ collisions in an impact energy range up to the $\mathrm{MeV}$ level, which largely encompasses the intermediate and high-energy regimes of interest for ion-based cancer therapy applications.

This research was supported by LaserLab Europe proposal MBI001886, Laserlab Charpac program (Grant Agreement No. 284464, EC's Seventh Framework programm), ELI-Beamlines (CZ.1.05/1.1.00/483/02.0061) and OPVK 3 (Grant No. CZ.1.07/2.3.00/20.0279), Transregio18 (DFG), and EPSRC grants EP/E035728/1, EP/F021968/1 and EP/K022415/1. P.V.N. acknowledges the support of World Class University Program (Grant No. R31-10026) funded by the Korean Ministry of Education (MOE).

\footnotetext{
${ }^{1}$ J. Ishikawa, H. Tsuji, M. Mimura, S. Ikemura, and Y. Gotoh, Surf. Coat Technol. 103/104, 173 (1998).

${ }^{2}$ J. Ishikawa, H. Tsuji, N. Arai, T. Matsumoto, K. Ueno, K. Adachi, H. Kotaki, and Y. Gotoh, Nucl. Instrum. Methods Phys. Res. B 237, 422 (2005).

${ }^{3}$ H. Tsuji, M. Izukawa, R. Ikeguchi, R. Kakinoki, H. Sato, Y. Gotoh, and J. Ishikawa, Nucl. Instrum. Methods Phys. Res. B 206, 507 (2003).

${ }^{4}$ H. Tsuji, M. Izukawa, Y. Utagawa, R. Ikeguchi, R. Kakinoki, H. Sato, Y. Gotoh, and J. Ishikawa, Trans. Mater. Res. Soc. Jpn. 29, 575 (2004).
} 
${ }^{5}$ Y. Takeiri, O. Kaneko, K. Tsumori, Y. Oka, K. Ikeda, M. Osakabe, K. Nagaoka, E. Asano, T. Kondo, M. Sato, and M. Shibuya, Nucl. Fusion 46, S199 (2006)

${ }^{6}$ Y. Ikeda, N. Umeda, N. Akino, N. Ebisawa, L. Grisham, M. Hanada, A. Honda, T. Inoue, M. Kawai, M. Kazawa, K. Kikuchi, M. Komata, K. Mogaki, K. Noto, F. Okano, T. Ohga, K. Oshima, T. Takenouchi, Y. Tanai, K. Usui, H. Yamazaki, and T. Yamamoto, Nucl. Fusion 46, S211 (2006).

${ }^{7}$ The European Spallation Source Study, The ESS Technical Study, report ESS-96-53-M, ISBN 0902376659 (1996). http://www.ess-europe.de.

${ }^{8}$ B. R. Appleton, J. B. Ball, and J. R. Alonso, The National Spallation Neutron Source EPAC'96 (Sitges, 1996), p. 575.

${ }^{9}$ J. John, C. P. Robinson, J. P. Aldridge, W. J. Wallace, K. R. Chapman, and R. H. Davis, Nucl. Instrum. Methods 57, 105 (1967); B. L. Donnally and G. Thoeming, Phys. Rev. 159, 87 (1967); R. M. Ennis, D. E. Schechter, G. Thoeming, and D. B. Schlafky, IEEE Trans. Nucl. Sci. NS14, 75 (1971); G. Phillipp, V. Scheib, and A. Hofmann, Nucl. Instrum. Methods 115, 507 (1974); E. B. Hooper, Jr., P. Poulsen, and P. A. Pincosy, J. Appl. Phys. 52, 7027 (1981).

${ }^{10} \mathrm{~N}$. Wells, The Development of High-Intensity Negative Ion Sources and Beams in the USSR, R-2816-ARPA (The Rand Corporation, 1981), p. 29, and references within.

${ }^{11}$ A. J. T. Holmes, Plasma Sources Sci. Technol. 5, 453 (1996).

${ }^{12}$ M. Bacal, Nucl. Fusion 46, S250 (2006).

${ }^{13}$ A. Ueno and K. Ikegami, Rev. Sci. Instrum. 75, 1714 (2004).

${ }^{14}$ G. D. Alton, Electrostatic Accelerators: Fundamentals and Applications, edited by R. Hellberg (Springer, Berlin, 2005), p. 222.

${ }^{15}$ S. Ter-Avetisyan, M. Schnürer, H. Stiel, and P. V. Nickles, J. Phys. D: Appl. Phys. 36, 2421 (2003).

${ }^{16}$ T. Nakamura, Y. Fukuda, A. Yogo, M. Tampo, M. Kando, Y. Hayashi, T. Kameshima, A. S. Pirozhkov, T. Zh. Esirkepov, T. A. Pikuz, A. Ya. Faenov, H. Daido, and S. V. Bulanov, Phys. Lett. A 373, 2584 (2009).

${ }^{17}$ S. Ter-Avetisyan, B. Ramakrishna, M. Borghesi, D. Doria, M. Zepf, G. Sarri, L. Ehrentraut, A. Andreev, P. V. Nickles, S. Steinke, W. Sandner, M. Schnürer, and V. Tikhonchuk, Appl. Phys. Lett. 99, 051501 (2011).
${ }^{18}$ R. Rajeev, T. M. Trivikram, K. P. M. Rishad, V. Narayanan, E. Krishnakumarand, and M. Krishnamurthy, Nat. Phys. 9, 185 (2013).

${ }^{19}$ R. Prasad, M. Borghesi, F. Abicht, P. V. Nickles, H. Stiel, M. Schnürer, and S. Ter-Avetisyan, Rev. Sci. Instrum. 83, 083301 (2012).

${ }^{20}$ S. Ter-Avetisyan, M. Schnürer, and P. V. Nickles, J. Phys. D: Appl. Phys. 38, 863 (2005).

${ }^{21}$ R. Prasad, F. Abicht, M. Borghesi, J. Braenzel, P. V. Nickles, G. Priebe, M. Schnürer, and S. Ter-Avetisyan, Rev. Sci. Instrum. 84, 053302 (2013).

${ }^{22}$ F. Abicht, R. Prasad, M. Borghesi, G. Priebe, J. Bränzel, A. Andreev, P. V. Nickles, M. Schnürer, S. Jequier, G. Revet, V. Tikhonchuk, and S. TerAvetisyan, "Energetic beams of negative and neutral hydrogen from intense laser plasma interaction," Appl. Phys. Lett. (submitted).

${ }^{23}$ C. Illescas, L. F. Errea, L. M'endez, B. Pons, I. Rabadán, and A. Riera, Phys. Rev. A 83, 052704 (2011); I. Kaganovich, E. Startsev, and R. C. Davidson, New J. Phys. 8, 278 (2006); B. M. Smironv, Usp. Fiz. Nauk. 171, 233 (2001).

${ }^{24}$ N. Bohr, K. Dan. Vidensk. Selsk. Mat. Fys. Medd. 18, 8 (1948).

${ }^{25}$ B. G. Lindsay, W. S. Yu, K. F. McDonald, and R. F. Stebbings, Phys. Rev. A 70, 042701 (2004).

${ }^{26}$ Atomic Data for Fusion, edited by R. A. Phaneuf, R. K. Janev, and M. S. Pindzola (US AEC Oak Ridge Natl. Lab, 1987), Vol. 5, p. 6090, http:// www-cfadc.phy.ornl.gov/redbook.

${ }^{27}$ M. M. Sant'Anna, W. S. Melo, A. C. F. Santos, G. M. Sigaud, and E. C. Montenegro, J. Phys. B 33, 353 (2000)

${ }^{28}$ Yu. A. Balkova, N. V. Novikov, and Ya. A. Teplova, J. Surf. Invest. 3, 188 (2009).

${ }^{29}$ T. V. Goffe, M. B. Shah, and H. B. Gilbody, J. Phys. B 12, 3763 (1979).

${ }^{30}$ I. T. Serenkov, R. N. II'in, V. A. Oparin, and E. S. Solov'ev, Sov. Phys. JETP 41, 845 (1976).

${ }^{31}$ H. H. Lo and W. L. Fite, At. Data 1, 305 (1970).

${ }^{32}$ J. O. Olsen and P. Hvelplund, J. Phys. B 7, 1331 (1974).

${ }^{33}$ T. Jorgensen, Jr., C. E. Kuyatt, W. W. Lang, D. C. Lorents, and C. A. Sautter, Phys. Rev. 140, A1481 (1965). 